\title{
Palmfarne im Palmengarten - eine einzigartige Nacktsamer-Gruppe
}

\author{
Veit M. Dörken \& Hilke Steinecke
}

\begin{abstract}
Palm ferns are ancestral seed plants and therefore often called living fossils. The Palmengarten holds a rich collection of palm ferns. Biology, ecology, culture and uses of palm ferns are described. Some species of the collection are described in more detail.
\end{abstract}

\section{Zusammenfassung}

Palmfarne sind eine ursprüngliche Gruppe von Samenpflanzen und werden daher oft auch als lebende Fossilien bezeichnet. Der Palmengarten beherbergt eine umfangreiche Palmfarn-Sammlung. Biologie, Ökologie, Kultur und Verwendung von Palmfarnen werden erläutert. Zudem werden einige markante Arten, die im Palmengarten kultiviert werden, erläutert.

\section{Einleitung}

Palmfarne gehören mit zu den eigentümlichsten heute lebenden Pflanzengruppen. In ihrer Wuchsform und mit den gefiederten, im jungen Stadium oft eingerollten Blättern erinnern sie habituell an Palmen und Farne, daher die deutsche Bezeichnung Palmfarne. Als Nacktsamer sind sie jedoch mit beiden Pflanzengruppen nicht näher verwandt, dafür aber mit den Koniferen. Palmfarne sind beliebte Zierpflanzen. In milden Regionen Europas wie z. B. im Mittelmeergebiet, auf den Kanaren oder auf Madeira zieren sie so manchen Straßenrand, öffentlichen Park oder auch privaten Garten und fehlen vermutlich in keinem Botanischen Garten. Über die Cycadeen-Sammlung im Botanischen Garten von Xalapa in Mexiko wurde in der vorhergehenden Ausgabe der Palmengarten-Zeitschrift berichtet (Schneider \& Mika 2012). Auch der Palmengarten beherbergt eine artenreiche Sammlung verschiedener Palmfarne, die in diesem Beitrag vorgestellt werden soll. Einige Palmfarne werden in der nicht öffentlich zugänglichen botanischen Sammlung kultiviert, andere sind in den Schauhäusern (vor allem im Tropicarium) ausgepflanzt. Dort haben sich ältere Pflanzen zu mittlerweile recht stattlichen

Abb. 1 (oben): Fossiles Blatt von Zamites feneonis aus Frankreich, rund 150 Mio. Jahre alt.

Abb. 2 (unten): Korallenwurzel im Längsschnitt von Ceratozamia mexicana.
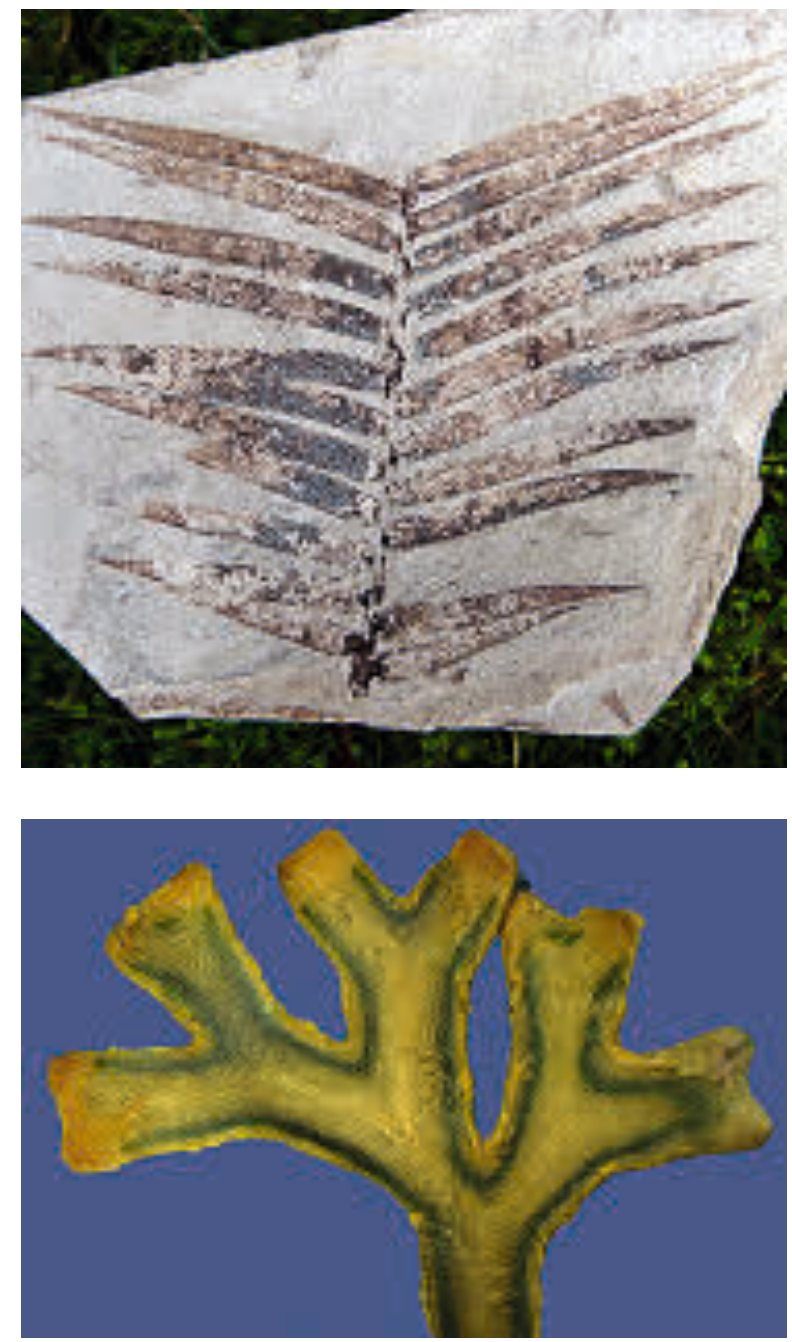

Individuen entwickelt. Im Sommerhalbjahr befinden sich einige als Kübelpflanzen kultivierte Palmfarne in der "Sommerfrische" im SommerSukkulentengarten. Manche Palmfarne wie z. B. Microcycas calocoma aus Kuba sind sehr selten und nur in wenigen Sammlungen vertreten. 


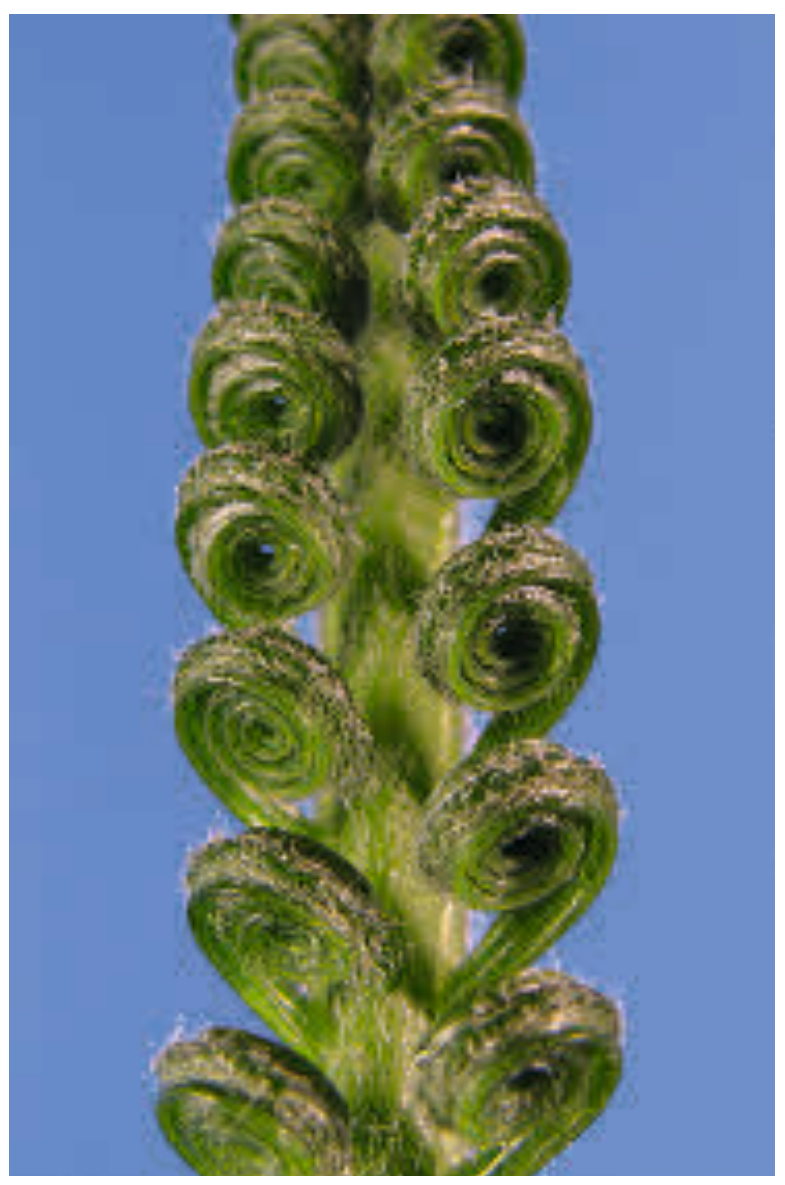

\section{Systematik und Verbreitung}

Die Palmfarne sind eine erdgeschichtlich sehr alte Gruppe. Sie traten nach Jones (1993) zuerst im Unterperm (vor 299-270 Mio. Jahren) auf. Wie Ginkgo werden sie als lebende Fossilien bezeichnet. Auf Fossilienbörsen werden gelegentlich versteinerte Blätter von Zamites-Arten (Jura, vor 200-146 Mio. Jahren) angeboten, die eine ziemliche Ähnlichkeit mit rezenten Palmfarnblättern aufweisen.

Mit 303 heute noch lebenden Arten aus zwei Familien (Cycadaceae, Zamiaceae) nehmen Palmfarne aktuell etwa ein Drittel der existierenden Nacktsamer ein. Die Cycadaceae umfassen nur die Gattung Cycas, die mit 98 Arten fast ein Drittel der heutigen Palmfarne ausmacht. Zu den Zamiaceae gehören 10 Gattungen: Encephalartos (65 Arten), Zamia (58 Arten), Macrozamia (40 Arten) und Ceratozamia (21 Arten) sind relativ artenreich, während die Gattungen Dioon (13 Arten), Bowenia, Chigua und Lepidozamia (mit jeweils nur $2 \mathrm{Ar}$ ten) deutlich artenärmer sind. Microcycas und
Stangeria sind monotypisch (Hill et al. 2004). Manche Systematiker stellen Stangeria und Bowenia in die eigenständigen Familien Stangeriaceae und Boweniaceae (JonES I993, МАBBERLEY 2008).

Zamiaceae kommen auf der Nord- und Südhalbkugel in Mittel- und Südamerika, Zentral-, Ost- und Südafrika sowie in Australien vor. Cycadaceae kommen in Ost-Afrika, Madagaskar, von Ost- über Südost-Asien bis Neukaledonien und Tongo vor (Stevens 2001). Die stark disjunkte Verbreitung beider Familien ist auf das Auseinanderdriften der Kontinente nach dem Auseinanderbrechen des Urkontinentes Pangaea zurückzuführen. Palmfarne waren ehemals wesentlich weiter verbreitet, als das heutige Verbreitungsmuster vermuten lässt (HARRIs 1961). Sie wuchsen auch in vielen Regionen, in denen heute keine Palmfarne mehr zu finden sind. Fossile Belege zeigen, dass Palmfarne ehemals auch in weiten Teilen Europas heimisch waren, so auch in Regionen des heutigen Deutschlands (Rozynek 2008). In fossilen Ablagerungen aus der Trias (vor 251-199,6 Mio. Jahren) konnten Palmfarne sogar in der Antarktis nachgewiesen werden (Hermsen et al. 2004).

\section{3. Äußeres Erscheinungsbild}

\subsection{Habitus}

Der Großteil der Palmfarne sind kleine Schopfbäume. Nur Zamia pseudoparasitica aus Panama wächst epiphytisch. Palmfarne haben einen auffällig dicken und gefeldert wirkenden Stamm mit einem stärkehaltigen Mark. Bei vielen Arten bilden die Wurzeln an der Erdoberfläche knollenartige Auswüchse (sog. koralloide

Abb. 3 (oben): Eingerolltes junges Blatt von Cycas revoluta.

Abb. 4 (Seite 107 oben): Pollenzapfen von Zamia pumila, ganz und quer.

Abb. 5 (Seite 107 Mitte): Rasterelektronenmikroskopische Aufnahme eines Mikrosporophylls von Zamia amblyphyllidia.

Abb. 6 (Seite 107 unten): Makrosporophyllstand von Cycas revoluta. 
Wurzeln). In diesen leben Luftstickstoff fixierende Bakterien aus den Gattungen Anabaeana, Calothrix und Nostoc.

\subsection{Blätter}

Palmfarne sind immergrün und entwickeln große, teilweise über drei Meter lange Fiederblätter, die wie bei Palmen schopfartig dicht gedrängt an der Spross-Spitze stehen. Bei einigen Arten sind die jungen Blätter wie bei Farnen eingerollt. Die Blätter der Cycadaceae haben eine auffällige Mittelrippe und einen glatten Blattrand, während bei den Zamiaceae eine deutliche Mittelrippe fehlt und der Blattrand leicht bis stark stechend gezähnt ist.

\subsection{Männliche und weibliche Fortpflanzungsorgane}

Alle Palmfarne sind diözisch. Es gibt also nur rein männliche und nur rein weibliche Individuen. Die männlichen Fortpflanzungsorgane sind bei beiden Familien in zapfenartigen, aufrechten Strukturen zusammengefasst. Diese zapfenartigen „Blüten“ umfassen zahlreiche spiralig stehende, Pollen produzierende Schuppen. Diese Mikrosporophyllen entsprechenden stark reduzierten Blätter und tragen auf ihrer Unterseite zahlreiche Pollensäcke (Gifford \& FosTER 1996). Die Mikrosporophylle stehen, wie es für echte Blätter typisch ist, nicht in der Achsel eines Tragblattes. Die Pollensäcke sind meist zu dritt miteinander zu einem Synangium vereint, das kurz gestielt sein kann. Studien zur Entwicklungsgeschichte der Palmfarne haben gezeigt, dass die Position der Synangien auf der Blattunterseite ein abgeleitetes Merkmal ist. Bei Vorfahren der heutigen Palmfarne waren sie vermutlich randständig angeordnet und wurden erst später auf die Unterseite verlagert; dieser Positionswechsel ist heute nur noch in ganz jungen Zapfen erkennbar. Somit konnte gezeigt werden, dass sich die Mikrosporophylle wie auch die Makrosporophylle von Fiederblättern ableiten lassen (Mundry \& Stützel 2004).

Bei den Zamiaceae stehen die weiblichen reproduktiven Strukturen ebenfalls in aufrechten, unverzweigten zapfenartigen Strukturen, die den Einzelblüten der Bedecktsamer entspre-
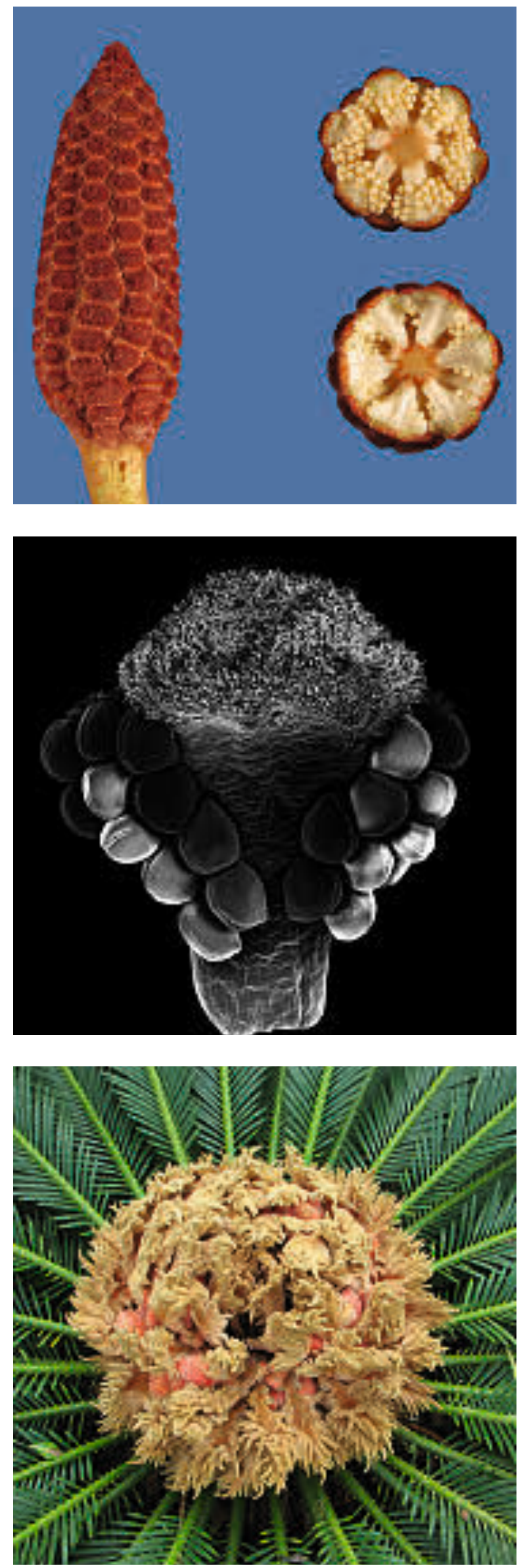

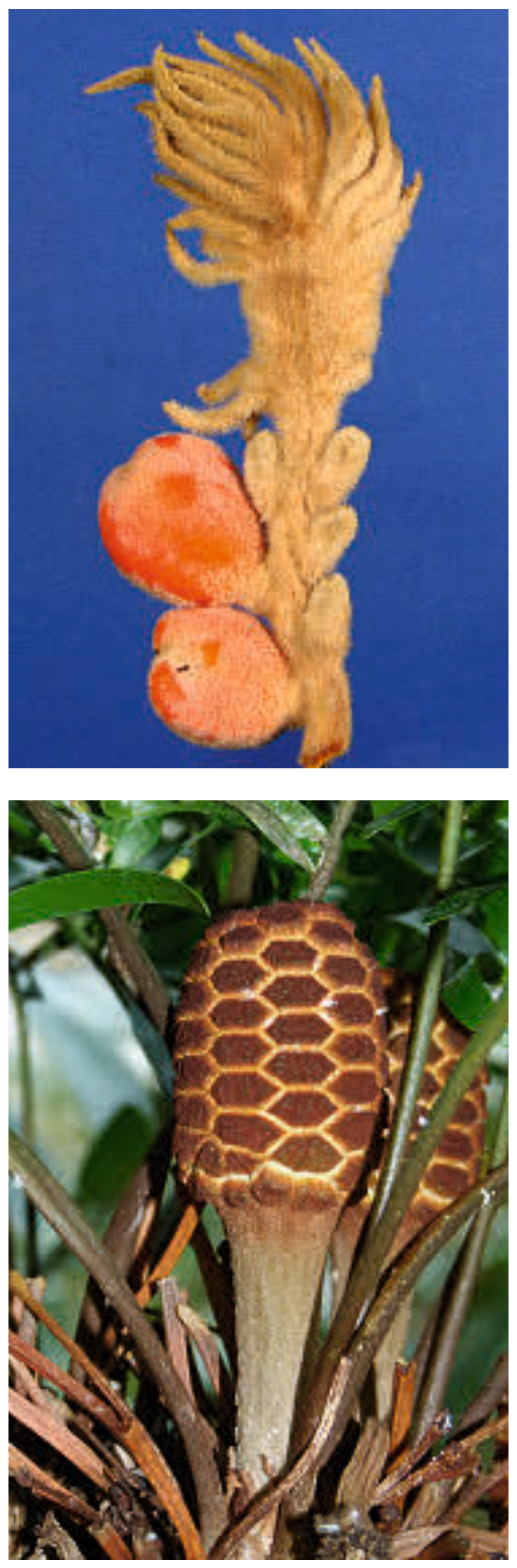

chen. Sie sind aus zahlreichen spiralig angeordneten und an der Spitze tischartig abgeflachten Makrosporophyllen (Samenblätter) aufgebaut. Das Makrosporophyll entspricht ebenfalls einem stark reduzierten, echten Blatt, das nur zwei Samenanlagen trägt, die im Inneren des Zapfens verborgen liegen. Die Mikropyle (die kleine Öffnung, durch die der Pollen in das Innere der Samenanlage gelangt) ist dabei zur Zapfenspindel hin orientiert. Zum Zeitpunkt der Bestäubung öffnet sich der Zapfen in Form eines kleinen Spaltes im terminalen und auch basalen Bereich. Die Bestäubung erfolgt überwiegend durch Käfer. Zamiaceae-Zapfen können je nach Art bis rund $60 \mathrm{~cm}$ lang und bis $40 \mathrm{~kg}$ schwer werden. Die Zapfen der Zamiaceae zerfallen zum Zeitpunkt der Samenreife. Es kommt dabei zur Austrocknung des Zapfens und damit verbunden zu starker Schrumpfung der Zapfenspindel. Da das tischartige Ende der Makrosporophylle nicht oder nur kaum schrumpft, werden die Makrosporophylle förmlich von der stark schwindenden Zapfenspindel abgerissen. Letztendlich bleibt nur noch die Zapfenspindel an der Pflanze erhalten, wie man dies auch z. B. von Tannen (Abies), Zedern (Cedrus) oder auch Araukarien (Araucaria) kennt.

Bei den Cycadaceae bildet der Vegetationspunkt des Sprosses in gewissen Abständen fiederblattartige, meist chlorophyllarme Makrosporophylle aus. Diese tragen im unteren Bereich des Blattes in der Position basaler Fiederblättchen randständig mehrere Samenanlagen. Im Gegensatz zu den zapfenartigen „Blüten“ der übrigen Palmfarn-Arten wird der Vegetationspunkt dabei nicht aufgebraucht, sondern bringt in der darauffolgenden Vegetationsperiode wieder grüne und gefiederte Laubblätter (Trophophylle, Ernährungsblätter) hervor.

Abb. 7 (oben): Makrosporophyll von Cycas revoluta mit zwei roten Samen.

Abb. 8 (unten): Weiblicher Zapfen von Zamia integrifolia.

Abb. 9 (Seite 109): Tischchenförmiges Makrosporophyll von Zamia amblyphyllidia mit zwei roten Samen. 
Die Mikropyle zeigt bei den Cycadaceae von der Blattspindel weg.

Die Samen haben eine Hülle (Integument), die sich mit zunehmender Reife in eine äußere fleischige, bei vielen Arten meist kräftig orange bis rot gefärbte Sarkotesta sowie eine innere stark verholzende Sklerotesta ausdifferenziert. Die Samen enthalten reichlich Alkaloide.

Palmfarne haben, wie auch Ginkgo, einen sehr ursprünglichen Befruchtungsmechanismus mit begeißelten und frei beweglichen Keimzellen (Spermatozoiden). Bei den übrigen Nacktsamern (den klassischen Nadelbäumen) sowie den Bedecktsamern erfolgt die Befruchtung der Eizellen mittels Pollenschlauch, d. h. es gibt keine frei beweglichen männlichen Geschlechtszellen mehr.

\section{Auswahl einiger Palmfarne im Palmengarten}

Palmfarne werden im Tropicarium sowohl in den Häusern der Trockenen Tropen sowie in den Feuchten Tropen gezeigt, wobei Arten aus beiden Familien (Cycadaceae, Zamiaceae) vorhanden sind.

$\mathrm{Zu}$ den trockenheitsliebenden Arten zählt z. B. die aus Australien (Queensland, New South Wales) stammende Lepidozamia peroffskyana. Sie wird im neuen Australienbeet im Savannenund Dornwälder-Haus gezeigt. Der Gattungsname bezieht sich auf den schuppigen Stamm (gr. lepidos = schuppig). Dieser stattliche Palmfarn entwickelt $3 \mathrm{~m}$ lange dunkelgrüne bis bläuliche Fiederblätter, die bis 250 einzelne Fiedern umfassen. Die männlichen, 50-60 cm langen und $20-30 \mathrm{~cm}$ breiten Zapfen sind lang gestielt. Die im Querschnitt viereckigen weiblichen Zapfen sind mit 30-60 cm Länge sowie 10-15 cm Breite deutlich kleiner als die männlichen. Jedes Makrosporophyll in den weiblichen Zapfen hat eine aufrechte kleine Spitze. Die Samen sind zum Zeitpunkt der Reife leuchtend rot gefärbt.

In direkter Nachbarschaft, auf dem Afrikabeet im Savannen- und Dornwälder-Haus, werden verschiedene Brotpalmfarne (Encephalartos) gezeigt. Sie sind in Zentral- und Südafrika heimisch. Arten der Gattung Encephalartos bilden

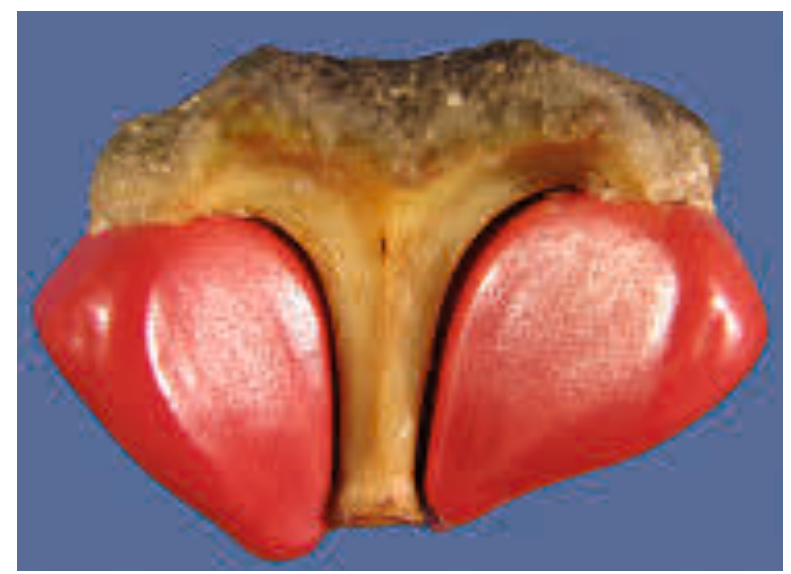

mitunter riesige Zapfen aus, die über Kopfgröße erreichen. Einer der ältesten Palmfarne des Gartens, der vermutlich noch aus der Sammlung des Herzogs Adolph von Nassau in Biebrich stammt, ist Encephalartos altensteinii. Die Pflanze wurde bereits in den alten Schauhäusern des Palmengartens kultiviert und später dann in das neue Tropicarium verpflanzt. Der Stamm wird bis $5 \mathrm{~m}$ hoch und $35 \mathrm{~cm}$ breit. Daneben wächst $E$. longifolius, ein mittelgroßer Palmfarn aus der östlichen Kap-Region. Sein kräftiger Stamm wird bis 4,5 m hoch, durch Blattnarben ist er gefeldert. An der Spitze bildet sich ein Schopf aus 1-2 m langen, Palmwedeln ähnlichen, stechenden Blättern. Das rund 50 Jahre alte Individuum hat 2011 erstmals einen weiblichen Zapfen gebildet, der mehrere Monate zum Auswachsen brauchte und dann noch etwa ein halbes Jahr an der Pflanze erhalten blieb, bis er dann schließlich zerfiel. Die Art gehört zu denjenigen Palmfarnen, die die größten weiblichen Zapfen bilden. Sie erreichen Ausmaße von 60 × $30 \mathrm{~cm}$ bei einem Gewicht bis zu $40 \mathrm{~kg}$. Encephalartos longifolius wurde von C. P. Thunberg und F. Masson erstmals um 1775 auf ihrer Reise nach Südafrika gesammelt und nach Europa gebracht. Die Art lässt sich leicht kultivieren. Die systematische Stellung von E. longifolius war lange Zeit umstritten, früher wurde die Art zur Gattung Zamia gestellt.

$\mathrm{Zu}$ den wohl spektakulärsten Zapfen eines Palmfarnes aus der Palmengarten-Sammlung gehören diejenigen von E. ferox. Das Exemplar im Eingangsoktogon bildet seit wenigen Jahren regelmäßig weibliche Zapfen. Die Zapfen bei- 

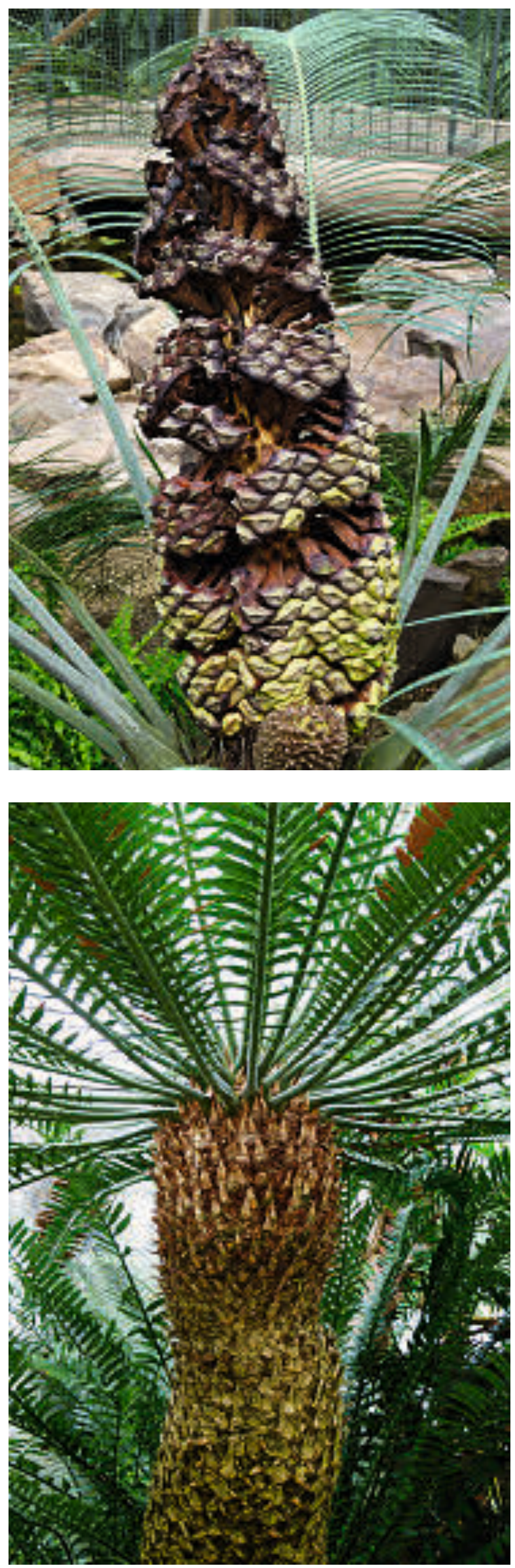

derlei Geschlechter sind intensiv ziegel- bis scharlachrot gefärbt und kontrastieren mit dem Dunkelgrün der Blätter. Die Samen sind leuchtend orange. Ebenfalls stark stechende Blätter (daher der Artbeiname) hat Encephalartos horridus. In Anpassung an den trockenen Lebensraum sind die Blätter von einer dicken Wachsschicht überzogen und dadurch blaugrau gefärbt. Die großen weiblichen Zapfen dieser Art sind olivgrün getönt. Mit mehreren Exemplaren gedeiht die aus der östlichen Kap-Provinz stammende Art im Dornwälder-Haus.

Manche Arten sind der Wissenschaft noch gar nicht so lange bekannt. So wurde die in nur einem sehr kleinen Areal des Lydenburg-Distrikts (Transvaal-Provinz, Süd-Afrika) heimische Art E. inopinus erst 1955 entdeckt (Jones 1993). Auch sie ist im Palmengarten vorhanden (Botanische Sammlung).

$\mathrm{Zu}$ den auffälligeren Palmfarnen des Palmengartens gehört die aus Mexiko (Sierra Madre) stammende Art Dioon edule var. edule, die Typusart der Gattung Dioon. Die wörtliche Übersetzung des Namens bedeutet "essbares Zwei-Ei" und bezieht sich auf die zwei eiförmigen, essbaren Samen pro Samenblatt. Ein junges, kleines männliches Individuum wurde erst vor wenigen Jahren neben das ältere weibliche Exemplar im Halbwüsten-Haus ausgepflanzt. Ihre bis $1,5 \mathrm{~m}$ langen Fiederblätter umfassen 100-150 grüne bis bläulich-grüne Fiederblättchen. Die weiblichen Zapfen sind zwar mit einer Länge bis $40 \mathrm{~cm}$ und einer Breite bis $20 \mathrm{~cm}$ nicht riesig, fallen aber durch ihre bräunliche, dicht-filzige Behaarung auf. Die beim Zerfallen des Zapfens anfallenden Einzelschuppen erinnern mit ihrer länglich zugespitzten Form und der fellartigen, weichen Behaarung an kleine Hasenohren. Die Spitze der Makrosporophylle ist stark rechtwinklig nach oben eingeknickt. Die männlichen zylindrischen Zapfen sind mit $30-40 \mathrm{~cm}$ Länge und 5-10 cm Breite deutlich

Abb. 10 (oben): Lepidozamia peroffskyana mit zerfallendem Pollenzapfen.

Abb. 11 (unten): Encephalartos altensteinii, einer der ältesten und größten Palmfarne im Palmengarten. 
kleiner. Die gelblichen Samen werden bis $3,5 \mathrm{~cm}$ lang.

Eine weitere aus Mexiko und Guatemala stammende und im Tropicarium kultivierte Art ist Ceratozamia robusta. Ein kräftiges, regelmäßig sehr große, männliche Zapfen bildendes Exemplar mit weit ausladenden Blättern steht im Savannen- und Trockenwälder-Haus seitlich der Brücke am Wasserfall. Vermutlich wegen des gestauchten, rundlichen und ähnlich wie eine Ananasfrucht gefelderten Stammes wird dieser Palmfarn auch Piña del Monte (Ananas des Berges) genannt. Die bis $3 \mathrm{~m}$ langen Fiederblätter setzen sich aus über 200 Fiederblättchen zusammen. Die weiblichen Zapfen sind mit $40-50 \mathrm{~cm}$ Länge und 10-15 cm Breite genauso groß wie die männlichen Zapfen. Bei allen Arten der Gattung Ceratozamia weist sowohl das Mikro- als auch das Makrosporophyll am distalen Ende zwei auffällige hornartige Auswüchse auf.

Eine beliebte und robuste Zimmerpflanze sowie eine der bekanntesten Palmfarne ist Zamia furfuracea. Der amerikanische Name Cardboard Palm bezieht sich auf die kartonartig steifen Blätter. Auch diese Art ist trockenheitsliebend, stammt aus Mexiko und wird im Halbwüsten-Haus gezeigt. Die Stämme bleiben meist gedrungen und die $30-100 \mathrm{~cm}$ langen jungen Fiederblätter sind wie die Zapfen dicht bräunlich behaart. Die weiblichen, bis $25 \mathrm{~cm}$ langen und bis $8 \mathrm{~cm}$ breiten Zapfen sind walzenförmig. Die Makrosporophylle sind am Ende tischartig abgeplattet. Mit bis $15 \mathrm{~cm}$ Länge und 2-3 cm Breite sind die männlichen Zapfen deutlich kleiner. Zur Reifezeit sind die Samen $2 \mathrm{~cm}$ breit und rosa bis rot gefärbt.

Auch verschiedene Vertreter der Gattung $C y$ cas (Sagopalmfarn) können im Tropicarium betrachtet werden. Im Dornwälder-Haus sind Exemplare von $C$. thouarsii zu sehen. Die Heimat

Abb. 12 (oben): Encephalartos longifolius mit weiblichem Zapfen.

Abb. 13 (unten): Encephalartos horridus.
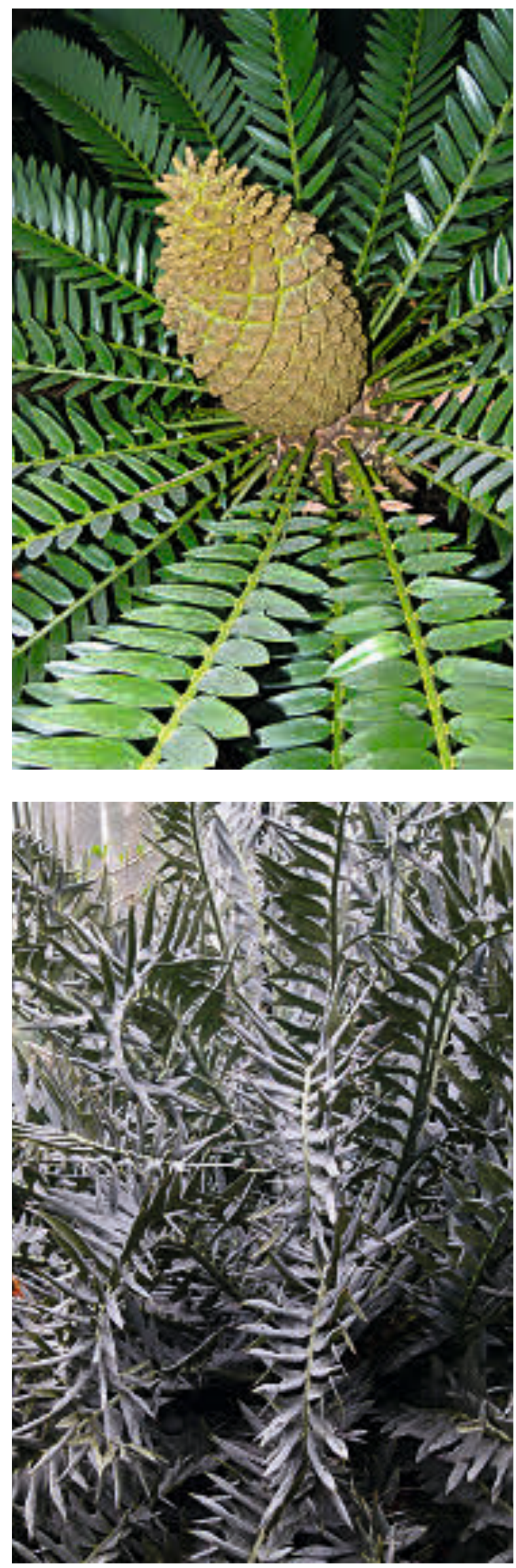


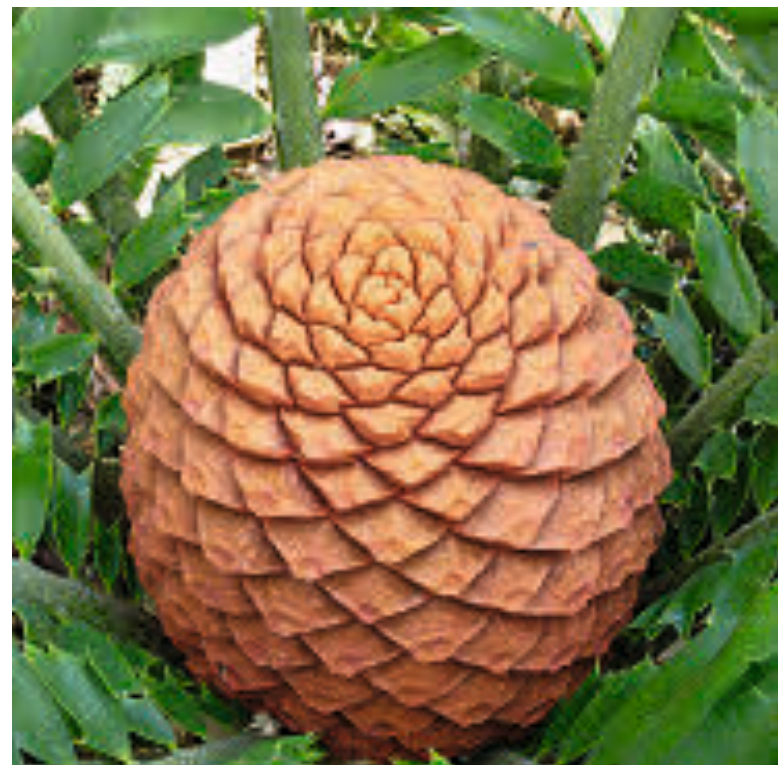

dieser Art liegt auf Madagaskar, den Seychellen und in Afrika (Mozambique, Tansania). CycasArten zeichnen sich durch auffällig große, gelbliche und teilweise riesige männliche Zapfen aus. So erreichen diese z. B. bei $C$. circinalis Längen von $40-60 \mathrm{~cm}$, bei $C$. revoluta 40 $70 \mathrm{~cm}$, bei $C$. rumphii $20-60 \mathrm{~cm}$ und bei $C$. thouarsii $40-70 \mathrm{~cm}$. Im Unterschied zu Arten der Zamiaceae sind die weiblichen Makrosporohphylle bei den Cycadaceae nicht in zapfenartigen Ständen angeordnet. Stattdessen werden Makrosporophylle (Samenblätter) und Trophophylle (Ernährungsblätter) an der Sprossachse in unregelmäßigen zeitlichen $\mathrm{Ab}$ ständen hervorgebracht. Es ist möglich, dass mehrere Jahre in Folge keine Samenblätter entstehen. Ältere Samenblätter sind auch nach Jahren an den Individuen zu erkennen, da sie nach Abwurf der reifen Samen nicht abfallen. Bei den meisten Arten sind die reifen Samen kräftig rot gefärbt, bei $C$. circinalis sind sie jedoch grünlich und stark bläulich wachsig bereift. Im Palmengarten sind Exemplare von C. revoluta und C. rumphii im Monsun- und PassatwälderHaus und im Mangroven- und KüstenwälderHaus zu sehen. Das bereits stattliche Exemplar von $C$. rumphii verdankt seinen Namen dem Naturforscher G. E. RumpF (geb. 1627 in Wölfersheim in der Wetterau in Hessen; 1702 gestorben auf den Molukken) und kann bis $4 \mathrm{Me}-$ ter hoch werden. Beide Arten sind mit beiden

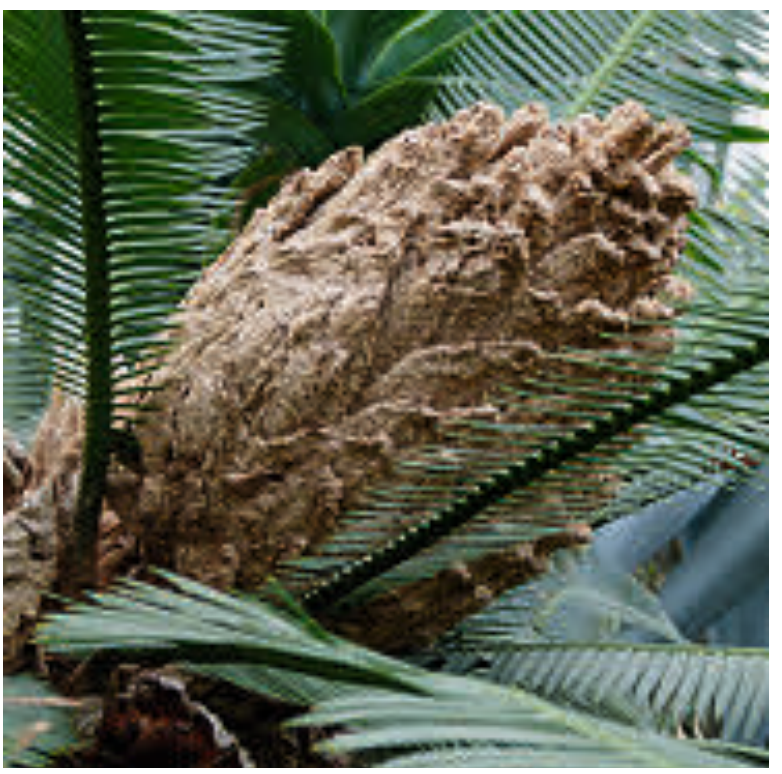

Geschlechtern vertreten und entwickeln regelmäßig männliche Zapfen sowie Samenblätter. Eine ganz besondere Cycas-Art (C. debaoensis) wird in der Botanischen Sammlung kultiviert. Sie zeichnet sich durch das für Palmfarne ungewöhnliche Merkmal doppelt gefiederter Blätter aus. Sie hat ein nur sehr kleines Verbreitungsareal in China (Region Debao im Westen der chinesischen Provinz Guangxi) und wurde erst 1996 entdeckt. Durch Pflanzenentnahme und Zerstörung des Lebensraumes sind die Bestände von $C$. debaoensis bedroht. In Kultur wächst dieser Palmfarn sehr schnell, entwickelt einen Schopf aus bis zu $3 \mathrm{~m}$ langen, glänzend grünen Blättern und bildet schon nach wenigen Jahren Zapfen und keimfähige Samen, so dass zunehmend legal vermehrte Individuen in den Handel kommen. Noch aber ist er in Sammlungen eine Besonderheit.

\section{Palmfarne in Kultur}

Palmfarne wachsen besonders gut, wenn sie ausgepflanzt sind und entsprechend viel Platz haben. $\mathrm{Zu}$ hohe Düngerkonzentrationen führen bei Palmfarnen zu einer Schädigung der

Abb. 14 (links): Ziegelroter weiblicher Zapfen von Encephalartos ferox.

Abb. 15 (rechts): Dioon edule mit weiblichem Zapfen. 
Wurzeln, weshalb mit Dünger eher sparsam umgegangen werden sollte. Besonders bei Topfkulturen ist darauf zu achten, dass keine Staunässe entsteht. Das Substrat sollte deshalb gut wasserdurchlässig, zudem leicht sauer, strukturstabil und salzarm sein. Es bewährt sich eine Mischung aus Bimskies und schlecht verrottbaren Dauerhumusstoffen (Holzkohle und Rindenmulch in unterschiedlicher Größe). Eine Düngung erfolgt während des Sommerhalbjahres regelmäßig mit etwa $1 / 4$ Promille Wuxal Typ K. Bei heißem Wetter werden die Topfkulturen 2-3 Mal wöchentlich kräftig mit lauwarmem Regenwasser gegossen. Im Winter (Raumtemperatur zwischen 12 und $16{ }^{\circ} \mathrm{C}$ ) wird nur etwa $1 \mathrm{Mal}$ wöchentlich gegossen. Beim Gießen werden die Pflanzen regelmäßig überbraust, was Staub sowie Schädlinge abspült. Bis zum nächsten Gießen sollte das Substrat zumindest an der Oberfläche wieder abgetrocknet sein. Im Sommer wird bei heißem Wetter die Topfsammlung zusätzlich regelmäßig morgens und mittags mit einem feinen Sprühgerät eingenebelt. Dioon-Exemplare benötigen mehr Wasser, da sie weniger Wasser als andere Palmfarne speichern können.

Palmfarne sind wie auch andere Gewächse durch Befall von Schädlingen gefährdet. Das beste Mittel, Krankheiten zu vermeiden, ist das Schaffen optimaler Kulturbedingungen und somit möglichst vitaler Pflanzen. Zamia furfuracea beispielsweise ist im Gewächshaus stets stark von Wollläusen befallen. Im Winter sind vor allem die Topfkulturen aufgrund von Lichtmangel und geringerer Stoffwechselaktivität schwächer und anfälliger, Verletzungen heilen nur schlecht aus. Im Sommer, wenn die Pflanzen im Freien stehen, werden sie vitaler und für Läuse unangreifbarer. Auch wenn der Standort sehr hell sein sollte, ist direkte Sonne zu vermeiden, da sonst die Blätter verbrennen können. Pflanzen, die im Frühsommer nach draußen

Abb. 16 (oben): Großer und stechender Samenzapfen von Ceratozamia robusta.

Abb. 17 (unten): Cycas rumphii; wenige Samenanlagen haben sich zu kugeligen Samen entwickelt.
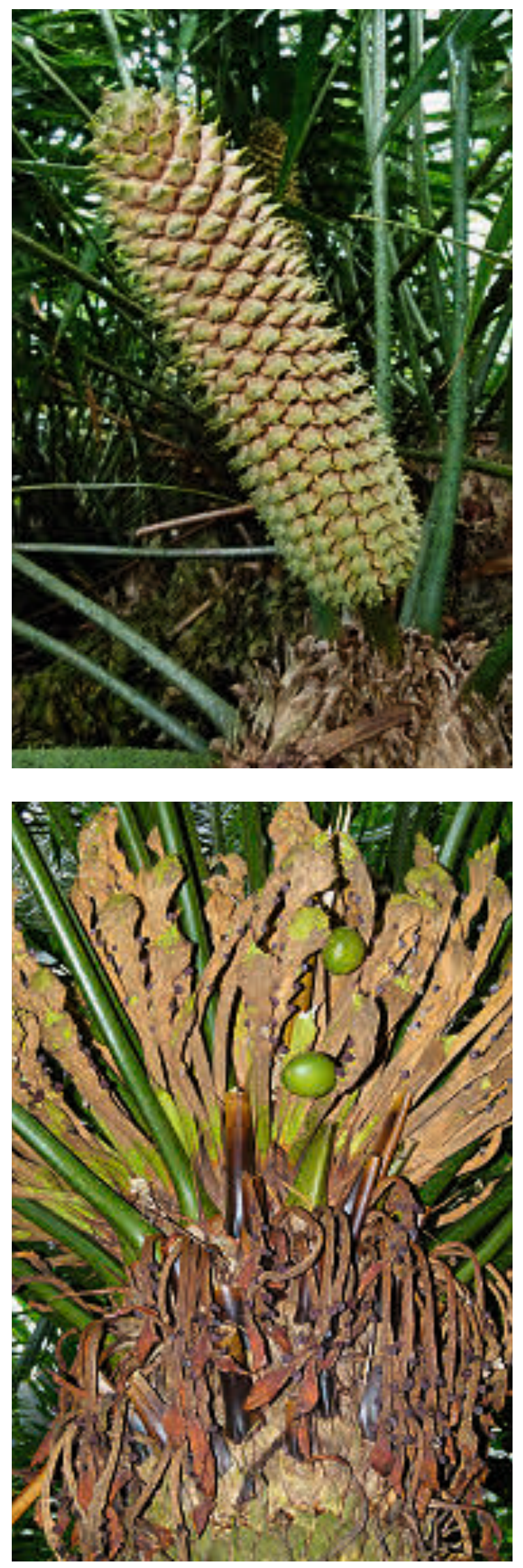


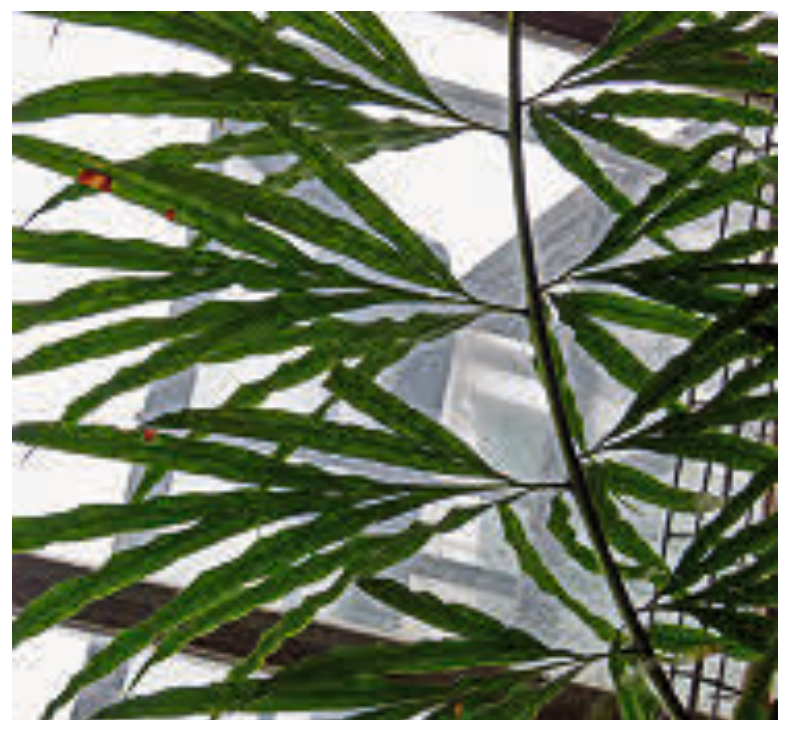

kommen, müssen erst an das starke Licht gewöhnt werden. Im Palmengarten wird bei den Cycadeen auf den Einsatz von Pflanzenschutzmitteln verzichtet. Stattdessen werden Nützlinge verwendet. Bei einem starken Befall von Wollläusen hilft das komplette Abschneiden der Wedel in Kombination mit nur geringen Wassergaben. Die Pflanzen treiben bald wieder aus.

In Gewächshauskultur lässt sich mit ein paar Tricks auch ein Samenansatz erzielen. Im Palmengarten werden zwischen die jungen Samenblätter von Cycas rumphii frische männliche Zapfen gehängt. Vermutlich durch Ameisen oder Fruchtfliegen erfolgt ein zufälliges Übertragen von Pollen auf einzelne Samenanlagen. Arten der Zamiaceae lassen sich leicht von
Hand bestäuben. Im Botanischen Garten Bochum hat sich folgende simple Bestäubungsmethode als sehr effektiv erwiesen: Hier werden die männlichen Pollenzapfen ab Februar regelmäßig auf Pollenreife hin überprüft. Dies geschieht durch leichtes Schütteln der Zapfen. Sobald sie anfangen auszustäuben, ist auch ihre Pollenreife erreicht. Zu diesem Zeitpunkt werden die Pollenzapfen dann geschnitten und kopfüber in einem Glasgefäß aufbewahrt. Meistens reifen die Samenzapfen zeitgleich mit den Pollenzapfen. Sollten die Samenzapfen zeitversetzt zu den Pollenzapfen heranreifen, können letztere auch kühl und trocken gelagert werden. Wenn mit dem Bestäuben begonnen werden soll, wird über die Pollenzapfen Wasser gegeben, so dass sich Pollen und Wasser gut vermengen. Dieses Gemisch wird dann reichlich mittels eines breiten Pinsels (mindestens Stärke 8 verwenden) in den oberen Spalt des geöffneten Zapfens gegeben. Diese Prozedur sollte täglich, am besten zur Mittagszeit und über einen mindestens dreiwöchigen Zeitraum, erfolgen.

\section{Nutzung}

Palmfarne sind traditionelle Stärkelieferanten, wobei Samen als auch Stämme verwendet werden. Vor allem aus den Stämmen von Cycas revoluta und $C$. circinalis wird falscher Sago gewonnen, während echter Sago ein Produkt der Sago-Palme (Metroxylon sagu, Arecaceae) ist. Sago wird zum Andicken von Suppen oder
Auf der kleinen, politisch zur USA gehörenden Pazifikinsel Guam tritt bei ungewöhnlich vielen Menschen eine tödlich verlaufende Nervenkrankheit mit Symptomen der Parkinson-Krankheit und der Alzheimerschen Krankheit auf. Sie wurde nach der Insel als Guam-ALS-PD-Demenzkomplex

(ALS/ PDC) bezeichnet. Indirekter Verursacher ist der auf der Insel heimische Brotpalmfarn $C y$ cas micronesica. Die Samen enthalten in geringen Mengen eine giftige Aminosäure (BMAA). Diese wird weder von Pflanzen noch Tieren produziert. Sie stammt von Nostoc-Blaualgen, die endosymbiontisch in den Cycadeen-Wurzeln leben. Die von den Blaualgen produzierten Aminosäuren werden von Cycas micronesica aufgenommen und im Pflanzenkörper sowie in den Samen verteilt. Die Samen werden von Flughunden gefressen, in denen sich die giftige Aminosäure anreichert. Die Flughunde gelten bei den Einheimischen als Leckerbissen, so dass sich auch bei den Menschen im Laufe der Zeit das Gift anreichert und es zur Erkrankung kommt. 
Kompott benutzt. Stärke aus Palmfarnen ist zudem Dickungsmittel für Puddings. Aber auch andere Palmfarnarten wurden früher lokal als Stärkelieferanten genutzt, worauf sich die Bezeichnung Brotpalmfarn für Arten der Gattung Encephalartos bezieht. Die Stärke wird oder wurde in manchen Gebieten zu einem alkoholischen Getränk vergoren. Um die vorletzte Jahrhundertwende wurde in Florida Palmfarnstärke aus Zamia integrifolia industriell gewonnen, wegen des Rückganges der Bestände jedoch wieder aufgegeben.

Blätter von Cycadeen sind in der Floristik beliebt und werden besonders in der Osterzeit als sog. Palmwedel genutzt. Vermutlich sind sie wegen ihrer Stabilität besser geeignet als Fiederblätter von echten Palmen.

Es ist reizvoll, eine umfangreiche Sammlung diverser Palmfarne aufzubauen, weil sie einerseits attraktive Zierpflanzen sind, anderseits als ursprüngliche Gewächse evolutionsbiologisch sehr interessant sind. Es ist aber zu beachten, dass sie unter das Washingtoner Artenschutzabkommen (CITES) fallen und bei der Anschaffung von Pflanzen auf die entsprechenden $\mathrm{Pa}$ piere zu achten ist.

\section{Dank}

Für kritische Durchsicht des Manuskriptes und Anregungen zu den Palmengarten-Cycadeen danken wir Kristina BaKhtiari-Kish und Martina Jacobi sowie Michael Goerigk (Bochum) für Infos zur Bestäubung von Palmfarnen in Kultur.

\section{Literatur}

Gifford, E. M. \& Foster, A. S. 1996: Morphology and evolution of vascular plants. 3. Aufl. - New York.

Harris, T. M. 1961: The fossil cycads. Palaeontology 4: 313-323.

Hermsen, E., Thomas, N., Taylor, T. N., Taylor, E. L. \& Stevenson, D. W. 2007: Cycads from the triassic of antartica: Perminaralized cycad leaves. - Int. J. Plant Sci. 168: 1099-1112.

Abb. 18 (Seite 114): Ausschnitt aus einem Wedel von Cycas daboeensis.

Abb. 19: Pollenzapfen von Cycas daboensis.

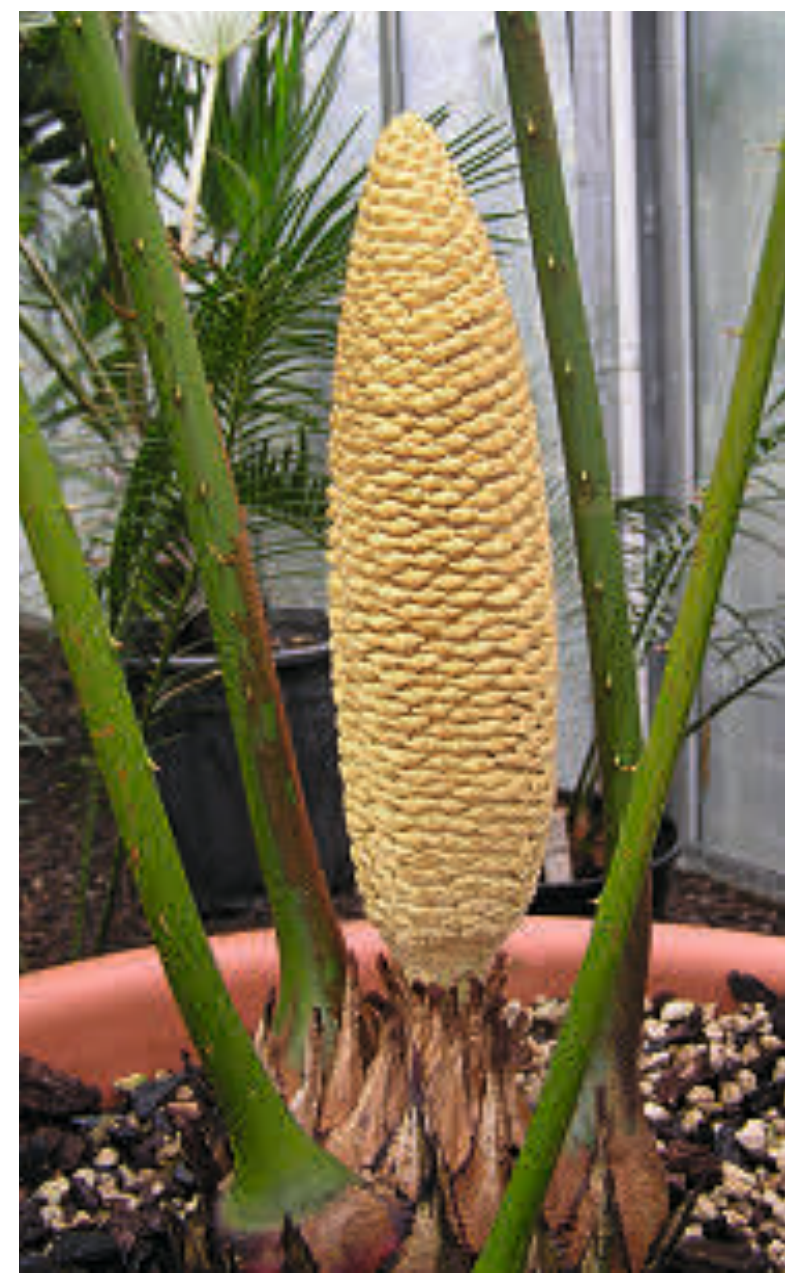

Hill, K. D., Stevenson, D. W. \& Osborne, R. 2004: The world list of cycads. In: Walters, T. \& Osborne, R. (Hrsg.) : Cycad classification: Concepts and recommendations. - Oxfordhire, Cambridge.

Jones, D. C. 1993: Cycads of the world. - Washington D. C.

Mabberley, D. J. 2008: Mabberley's plant book. 3. Aufl. - Cambridge.

Mundry, M. \& Stützel, T. 2003: Morphogenesis of male sporangiophores of Zamia amblyphyllidia STEV. - Plant. Biol. 5: 297-310.

RozyneK, B. 2008: Schozachia donaea n. gen., n. sp., a new cycad megasporophyll from the middle triassic (ladinian) of south germany. - Palaeodiversity 1: 1-17.

Schneider, J. V. \& Mika, M. 2012: Mexikanische Cycadeen und deren Schutzsammlung im Botanischen Garten von Xalapa, Veracruz, Mexiko. - Palmengarten 76: 5-11.

\section{Internetseiten}

http://m.schuelerlexikon.de/bio_abi2011/

Die_raetselhafte_Krankheit_von_Guam.htm http://www.mobot.org./MOBOT/research/APweb.: Stevens, P.F. : Angiosperm phylogeny website. Version 12, 12, July 2012. 